\title{
Generalized Linear Mixed Models can detect unimodal species-environment relationships
}

Niche theory predicts that species occurrence and abundance show non-linear, unimodal relationships with respect to environmental gradients. Unimodal models, such as the Gaussian (logistic) model, are however more difficult to fit to data than linear ones, particularly in a multi-species context in ordination, with trait modulated response and when species phylogeny and species traits must be taken into account. Adding squared terms to a linear model is a possibility but gives uninterpretable parameters.

This paper explains why and when generalized linear mixed models, even without squared terms, can effectively analyse unimodal data and also presents a graphical tool and statistical test to test for unimodal response while fitting just the generalized linear mixed model. R-code for this is supplied in supplementary information. 
2 Tahira Jamil ${ }^{1,2}$ and Cajo J. F. ter Braak ${ }^{1, *}$

$4 \quad{ }^{1}$ Biometris, Wageningen University and Research Centre, Box 100, 6700 AC Wageningen,

5 the Netherlands.

$6 \quad{ }^{2}$ Department of Mathematics, COMSATS Institute of Information Technology, Park Road,

7 Islamabad, Pakistan

$8 \quad{ }^{*}$ corresponding author. Tel.:+31 317480803 Fax +31 317483554 E-mail address:

9 cajo.terbraak@wur.nl

10 


\section{Introduction}

Niche theory predicts that species occurrence and abundance show non-linear, unimodal relationships with respect to environmental gradients (Austin 1987; Palmer \& Dixon 1990; Whittaker 1967). Many studies fail to test for unimodal response (Austin 2007). Thus straight-line relationships are often fitted without justification (e.g. Gibson et al.(2004) ). Pollock et al. (2012) propose a generalized linear mixed model for investigating trait modulation of the environmental response of a number of species. In their data unimodal response was said to be precluded, presumably as they examined relatively short environmental gradients. But might their method or a small modification thereof have worked for unimodal response? Ives \& Helmus (2011) recently proposed phylogenetic generalized linear mixed models. Can these models usefully analyze unimodal response?

A similar question arises in community ecological ordination, a class of multivariate methods to analyze the occurrence and/or abundance of a set of species in a set of sites and resulting in a configuration of the sites in a factorial plane, the directions of which can be interpreted as latent environmental variables (Jongman et al. 1995; ter Braak \& Prentice 2004; Walker \& Jackson 2011). Principal component analysis and redundancy analysis are linear ordination methods whereas (detrended) correspondence analysis and canonical correspondence analysis are claimed to be able to analyze unimodal response (ter Braak 1985; ter Braak 1986).

Nevertheless, (canonical) correspondence analysis is an eigen vector method and therefore inherently linear. This is most apparent in the reconstitution formula of (canonical) correspondence (Greenacre 1984; ter Braak \& Verdonschot 1995). How can it be understood that these methods are able to model unimodal data but are inherently linear? 
Some insight in this question is given by Ihm \& van Groenewoud (1984) and further worked out by ter Braak (1987) and de Rooij (2007) who showed the relation between the unimodal model and a generalized (bi)linear model, also known as Goodman's RC model. The relation can be used both ways. Ihm \& van Groenewoud (1984) use the relationship to justify their model B which is a (bi)linear model), for ecological ordination and de Rooij (2007) uses it to transform the linear predictor of the RC model into a quadratic form, with the graphical purpose to transform a vector representation or biplot to a distance representation that is supposed to be easier to interpret for naïve users of multivariate methods. Additional insight is given by Zhu et al.(2005) who introduced a weighted sample model to show the equivalence of canonical correspondence analysis and linear discriminant analysis.

In this paper we propose a graphical tool and statistical test to test for unimodal response while fitting just a generalized linear mixed model (GLMM) without squared terms. GLMMs are model-based, inferential statistical tools. GLMMs are very useful for describing the community patterns and are becoming popular in ecological and evolutionary studies (Bolker et al. 2009; Ives \& Helmus 2011; Zuur et al. 2009). Even when unimodality is detected using the proposed tool and test, we claim that GLMMs can effectively analyze unimodal data when the niche width is not very different among species and illustrate this claim by comparing the GLMM approach with an explicit unimodal model approach on data that show unimodal response. We focus on explicit environmental variables. In this case of direct gradient analysis we can of course add the squares of environmental variables to the model (ter Braak \& Looman 1986) and test the statistical significance of the addition, but the argument extends to the trait modulated response (Jamil et al. 2012) and to latent variable models (Walker \& Jackson 2011) for which models without squared terms are easier to handle with software that is more widely available. 
61

62 63

\section{Material \& Methods}

Generalized linear mixed models and unimodal response

For ease of exposition we start with a logistic linear mixed model for presence-absence data as example GLMM. The same approach can be followed for count data and loglinear models, which would relate to the RC model (de Rooij 2007). Consider the logistic linear mixed model that relates the probability of occurrence $p_{i j}$ of species $j$ in site $i$ to a quantitative

environmental variable $x_{i}$ by the formula

$$
\operatorname{logit}\left(p_{i j}\right)=\alpha_{j}+\beta_{j} x_{i}+\gamma_{i} \quad(i=1, \ldots, n ; j=1, \ldots, m) .
$$

with $\alpha_{j}$ an intercept, $\beta_{j}$ a slope and $\gamma_{i}$ a site effect, which we all take as random parameters with normal distributions with zero mean and variances $\sigma_{\alpha}^{2}, \sigma_{\beta}^{2}$ and $\sigma_{\gamma}^{2}$. In this random intercept, random slope model (Gelman \& Hill 2007; Zuur et al. 2009) it is prudent to have an additional parameter $\rho$ for the correlation between the intercepts $\alpha_{j}$ and slopes $\beta_{j}$; otherwise the model would change by just centering the environmental variable. Inclusion of the random site effects $Y_{i}$ are a means to avoid pseudoreplication (Hurlbert 1984) as they introduce correlation among species. These correlations were not modelled by Pollock et al. (2012) which makes their statistical tests liberal. The site effects may account for the size of the site, the fertility of the site or any other unknown factors that influence the probability of occurrence of all species in the site. The site effect $\gamma_{i}$ will thus 
80 be expected to be related to the expected number of species in a site, that is to $\sum_{j} p_{i j}$ and,

81 in terms of the data, to the number of species that is observed in a site, for short the site total,

82 defined as $S_{i}=\sum_{j} y_{i j}$. The site total and the site effect are expected to have a monotonic

83 positive relationship.

84 We now turn to one of the simplest unimodal curves for presence-absence, the Gaussian

85 logistic curve (Oksanen et al. 2001; ter Braak \& Looman 1986)

$86 \operatorname{logit}\left(p_{i j}\right)=a_{j}-\frac{\left(x_{i}-u_{j}\right)^{2}}{2 t_{j}^{2}}$

87 with $a_{j}$ a coefficient related to maximum probability of occurrence, $u_{j}$ the species

88 optimum and $t_{j}$ the tolerance of species $j$. This model has thus a logistic form but is

89 nonlinear in this parameterization. By expanding the quadratic term,

90

$$
a_{j}-\frac{\left(x_{i}-u_{j}\right)^{2}}{2 t_{j}^{2}}=a_{j}-\frac{1}{2 t_{j}^{2}} x_{i}^{2}-\frac{1}{2 t_{j}^{2}} u_{j}^{2}+\frac{1}{t_{j}^{2}} x_{i} u_{j}=\left(a_{j}-\frac{1}{2 t_{j}^{2}} u_{j}^{2}\right)+\left(\frac{u_{j}}{t_{j}^{2}}\right) x_{i}-\frac{1}{2 t_{j}^{2}} x_{i}^{2}
$$

91 it can be fitted to data of each individual species by a generalized linear model (GLM) by

92 using $x$ and $x^{2}$ as predictors (Jongman et al. 1995; ter Braak \& Looman 1986). The fit is

93 unimodal if the regression coefficient of $x^{2}$ is negative.

94 Might we be able to model unimodal response even without the squared term? On assuming

$95 \quad t_{j}=t$ and setting

$96 \quad \alpha_{j}=a_{j}-\frac{1}{2 t^{2}} u_{j}^{2}, \quad \beta_{j}=\frac{u_{j}}{t^{2}}$ and $\gamma_{i}=\frac{-1}{2 t^{2}} x_{i}^{2}$, 
97

98 not exactly hold because $x_{i}^{2} / t_{j}^{2}$ then also depends on $j$. With equal tolerances, unimodal

99

100

101

102

103

104

105

106

107

108

109

110

111

112

113

114

115

116

117

we obtain the equation (1) again. If $t$ would vary among species then equation (1) does

response can thus be represented by a simple linear model with site effects and, as we

propose, be fitted by a GLMM based on equation (1). The GLMM has additional normality

assumptions. In case of unimodal response, the assumption that the site effects $\left\{\gamma_{i}\right\}$ are

independent normal is false, as the site effects then depend on $x_{i}$ through $\left(x_{i}^{2}\right) / t^{2}$. This will be the basis of our test on unimodal response in the next section. The site effects in equation (4) also have a nonzero mean, but that is not a problem, as the mean can be taken

out and transferred to the intercepts $\alpha_{j}$.

The unimodal model with two or more environmental variables (ter Braak \& Prentice 1988)

can similarly be rewritten as a simple linear model without squared terms if the tolerances are equal (see Appendix). In conclusion, up to distributional assumptions, the GLMM (e.g. equation (1)) can be interpreted as a Gaussian logistic model with equal tolerances for the species.

\section{A graphical tool and statistical test for unimodal response}

Equation (4) suggests a graphical tool for detecting unimodal response and also a statistical test. The idea is to fit a GLMM to the binary data $\left\{y_{i j}\right\}$ with respect to the environmental variable with values $\left\{x_{i}\right\}(i=1, \ldots, n)$. In the R package lme4 (Bates et al. 2011), the model can be fitted by

$\operatorname{lmer}(y-1+x+(1+x \mid s p)+(1 \mid$ site $)$

$$
\text { family=binomial (link="logit") , data), }
$$


where y represents the vectorized response data while $s p$ and site are factors indicating species and sites. The site effects $\gamma_{i}$ obtained from the fit are then plotted against the environmental variable $\left\{x_{i}\right\}$. There is an indication of unimodal response in terms of the species response with respect to the environmental variable $x$ if this graph shows a n-shaped (as opposed to u-shaped) quadratic relationship. If the shape is not quadratic but curved, a transformation of x may improve it.

In the statistical test for unimodal response, the null model is the GLMM of equation (1) with, specifically, independent and normally distributed site effects. The alternative model is that the site effects depend quadratically on the environmental variable. As the site effects typically also depend on the site total $S$, a sensitive test on unimodal response is obtained by regressing the site effects on $x, \quad x^{2}$ and $S$, according to the model formula

$$
Y \sim x+x^{2}+S .
$$

There is evidence of unimodal response if the squared term is significant as judged by a z-test or, equivalently, an anova test on its regression coefficient, the null model being $Y \sim$ $x+S$. R-code for making the graph and performing the test on unimodality is supplied in supplementary information.

\section{Simulated data}

In the first example series the procedure to simulate data is the following:

1) Generate $n=50$ values of an environmental variable $x$ as a random sample from the 
2) Generate a vector $u$ of length $m$ from a uniform distribution $U(-\tau, \tau)$, where

$\tau=2+t$, for a fixed value of $t$, to ensure that optima are also placed outside the sample range of $x$.

3) Generate a vector $a$ of length $m$ drawn at random from the standard normal distribution.

4) Generate binomial probabilities from the unimodal response curve

$$
p_{i j}=\operatorname{logit}^{-1}\left(a_{j}-\frac{\left(x_{i}-u_{j}\right)^{2}}{2 t_{j}^{2}}\right)
$$

where $y_{i j}$ presence-absence data were generated at random from a Bernoulli distribution

with probability $\quad p_{i j}$ and $\quad t_{j}=t$. We simulate data with constant tolerance in each data set for $m=100$ species and vary ${ }^{t}$ between data sets ( $t=0.5,1$ and 4). Fig. 1 indicates how the simulated species response curves look like in each data set. We repeated the example with $x$ and $u$ having normal distributions instead $\left(x \sim N(0,1)\right.$ and $u \sim N\left(0, t^{2}\right)$ ), but do not show the results as they were very similar to the uniformly distributed case. In the second example series, we vary the tolerance lognormally among species, $t \sim$

$$
\operatorname{LogN}(0, \sigma) \text { with } \quad \sigma=0.25,0.5 \text { and } 1 \text { so that the median tolerance is 1. For } \sigma=1 \text {, }
$$
the coefficient of variation is larger than $100 \%$. In the third example series we vary the number of species ( $m=10,50$ and 100) with $t^{t}=1$. For the rest the simulation is set up as 
in the first series. We also simulated data according equation (1) with the strict assumptions of the GLMM of independent normal site effects.

Each dataset was characterized by beta diversity and length of gradient. The index of beta diversity is $\quad \beta_{w}=T / S-1$, where $T$ is the total number of species, and $S^{\prime}$ is the average number of species per site (Whittaker 1960). Length of gradient, obtained by analyzing the data with detrended correspondence analysis (DCA), is expressed in standard deviation (S.D.) units (Hill \& Gauch 1980). Values greater than 4 are commonly taken to indicate unimodal response. Beta diversity was calculated using the asbio package (Aho 2011) and DCA was performed in the vegan package (Oksanen et al. 2011), both in R.

\section{Real data}

We illustrate our method with three real data sets. The first is the Dune Meadow data (Jongman et al. 1995). This is a small data set of 28 higher plants in 20 sites in a dune area in the Netherlands. Environmental variables, related to soil and management, were measured at each site; we use the variable Moisture.

The second data set is about the vegetation of the rising seashore on the island Skabbholmen in the Stockholm archipelago, eastern central Sweden (Cramer \& Hytteborn 1987) and is part of the Canoco package (ter Braak \& Smilauer 1998). The data set consists of 63 sites sampled in both 1978 and 1984 and contains 68 species. The environmental variable is Elevation.

The third data set involves phytoplankton communities of 203 lakes located within four climate zones and associated measurements on various environmental variables and morphological species traits of 60 species (Kruk et al. 2011). We consider the environmental variables Temperature. 
For each data set we fit a GLMM according to equations (1) with $x$ the noted environmental variable, plot the resulting site effects against $x$ and test for unimodal response at the $5 \%$ significance level explained by and below equation (5). For the sea-shore data, we analyze years 1978 and 1984 separately. We also compare the regression coefficient $\beta_{j}$ as estimated by GLMM with the optimum $u_{j}$ as obtained by explicitly fitting equation (2) using GLM (ter Braak \& Looman 1986) for species with a well-defined optimum, that is, the squared term of which has a z-ratio smaller than -1 in the GLM model. In the small Dune Meadow data set we use z-ratio $<0$.

\section{Results}

\section{Simulated data}

Fig. 1 shows the simulated response curves in example series 1 ; the sampled range of the environmental variable is the range of $x$ shown. With increasing tolerance the part of the curves that is sampled shows less unimodal response. This is expressed quantitatively in the length of gradient SD units which varies between about 1 SD (not so unimodal) to 6 SD (very unimodal); the beta diversity varies correspondingly between 1 and 5 .

In Fig. 2, the site effects estimated by the GLMM analysis of each of the simulated data sets are plotted against the environmental variable. In all three series, site effects shows a clear quadratic relationship with the environmental variable except for large tolerance ( ${ }^{t=4}$ ) in the first series. Note the decreasing range of sites effects as the tolerance increases in the first row of Fig. 2; for large tolerance, the site effects are close to zero. Nevertheless, the squared term in equation (5) was significant in all examples $(\mathrm{P}<0.001)$ so that the method detects 
unimodal response even if it is moderate $(t=4)$. When the data are simulated according to equation (1) with the strict normality assumptions of the GLMM, the squared term was not judged significant more often than expected on the basis of Type I error of the test.

Fig. 3 shows the relationship between the random slopes ( $\beta_{j}$ ) estimated by GLMM and the true optima ( $u_{j}$ ) in the three simulation series. The relation is positive as predicted by equation (4). The relationship is weaker the larger the tolerance (Fig. 3, first row). With tolerance varying across species, the relationship continues to hold true surprisingly well (Fig 3, second row), except perhaps when the coefficient of variation of the tolerance is large $(>100 \%)$. The larger the number of species the clearer the predicted relationships (last row of Figs 2 and 3).

\section{Real data example}

The site effects estimated by GLMM show a quadratic relationship with the noted environmental variable in each of the three data sets (Fig. 4 top row), with the least unimodal response in the Dune Meadow data. Unimodal response is significant in all cases, as judged by our proposed significance test $(\mathrm{P}<0.001)$ and the relationship between the random slopes (

$$
\beta_{j} \text { ) estimated by GLMM and the optima ( } u_{j} \text { ) obtained from a fit of the unimodal }
$$
model of equation (1) is close to linear (Fig. 4, bottom row). In the Dune Meadow data there is one outlier for a species with a z-ratio close to 0 . In the phytoplankton data, the species with similar low values for the optimum received differential values for the slope, but otherwise there is a good agreement. 
$240 \quad \operatorname{logit}\left(p_{i j}\right)=a_{j}-\frac{\left(x_{i}-u_{j}\right)^{2}}{2 t^{2}}+\tilde{\gamma}_{i}$

$241 \quad$ with $t=1 / \sqrt{-2 \beta_{2}}, \quad u_{j}=t^{2} \beta_{j}, \quad a_{j}=\alpha_{j}+\frac{1}{2 t^{2}} u_{j}^{2} \quad$ and $\quad \tilde{\gamma}_{i}=\gamma_{i}+\frac{1}{2 t^{2}} x_{i}^{2}$.

242 We can go one step further and test the assumption of equi-tolerance by adding the squared 243 term $x^{2}$ also as a random (species-dependent) component to equation (7) and testing the 
significance of this extra variance component. In the R package lme4 (Bates et al. 2011), the two models to compare are (with $\mathrm{xx}=\mathrm{x} 2$ )

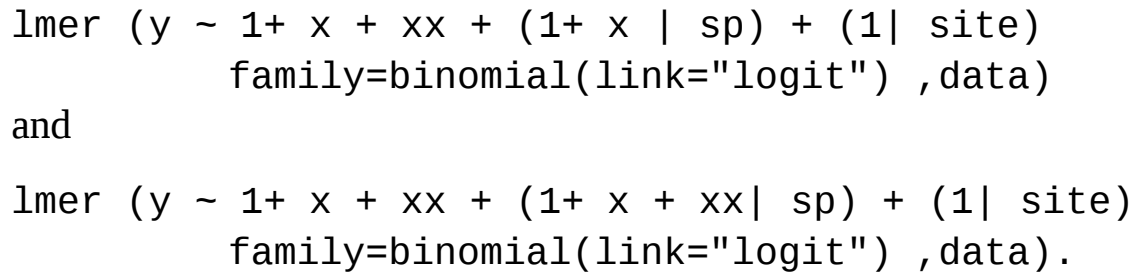

We are currently investigating robust ways of testing the equi-tolerance assumption on the basis of these two models, as the default ANOVA test has inflated type I error for small $t$.

The second way of interpretation is to conclude that the GLMM based on equation (1) is remarkably robust to the normality assumption on the site effects and the equi-tolerance assumption and that it can be used as a basis of more complicated models, such as the trait modulated response model (Jamil et al. 2012; Pollock et al. 2012) and latent variable models (Walker \& Jackson 2011), even for unimodal response. In this way, the site effects are treated as nuisance parameters and the independence and normal distributions, needed for efficient computation, as a prior distribution that a posteriori may turn out to be false. Cormont et al (2011) used this type of rationale to claim that their linear trait-environment method is well suited to analyse unimodal response.

\section{Conclusions}

Site effects in multi-species GLMM serve three purposes in ecological data, first to avoid pseudoreplication (Hurlbert 1984), second to account for differences in species richness among sites (Ives \& Helmus 2011) and third (this paper) to allow for common nonlinear, unimodal response. The results of this paper imply that the phylogenetic generalized linear mixed models of Ives \& Helmus (2011) are not in conflict with niche theory as they include random site effects and can thus deal with unimodal response. 
Walker \& Jackson (2011) used a latent variable approach to test for unimodal response. We tried their approach with the phytoplankton data, but failed to get an answer because the program for fitting the quadratic model crashed. We focussed this paper on the easier task of detection unimodal response to measured environmental variables by using a GLMM without squared terms. To scale up to a latent variable approach we need factor analytic structure within a GLMM. This already exists for linear mixed models (Thompson et al. 2003; Verbyla et al. 2003) and it is a matter of time that it becomes standardly available for GLMM. This paper shows the utility of such factor analytic models in ecology (Walker \& Jackson 2011) if they allow additional random site effects.

A GLMM with terms that are linear in quantitative predictors is, of course, linear. But with random site effects included, GLMMs can detect and fit unimodal response, with the provision that the differences in niche widths among species is not too large. The application scope of GLMM in ecology is thus much broader than one might think at first glance.

\section{Acknowledgements}

This paper benefited from input from Mike Palmer, Steve Walker and Mu Zhu.

\section{References}

Aho K. 2011. asbio: A collection of statistical tools for biologists. R package version 0.3-36. http://CRANR-projectorg/package=asbio.

Austin M. 2007. Species distribution models and ecological theory: A critical assessment and some possible new approaches. Ecological Modelling 200:1-19.

Austin MP. 1987. Models for the analysis of species' response to environmental gradients. Plant Ecology 69:35-45. 
Bates D, Maechler M, and Bolker B. 2011. lme4: Linear mixed-effects models using S4 classes. R package version 0.999375-39,. http://cranr-projectorg/web/packages/lme4/indexhtml.

Bolker BM, Brooks ME, Clark CJ, Geange SW, Poulsen JR, Stevens MHH, and White JSS. 2009. Generalized linear mixed models: a practical guide for ecology and evolution. Trends in Ecology and Evolution 24:127-135.

Cormont A, Vos CC, van Turnhout CAM, Foppen RPB, and ter Braak CJF. 2011. Using life-history traits to explain bird population responses to increasing weather variability Climate Research 49:59-71.

Cramer W, and Hytteborn H. 1987. The separation of fluctuation and long-term change in vegetation dynamics of a rising seashore. Plant Ecology 69:157-167.

de Rooij M. 2007. The distance perspective of generalized biadditive models: scalings and transformations Journal of Computational and Graphical Statistics 16 210-227.

Gelman A, and Hill J. 2007. Data Analysis Using Regression and Multilevel/Hierarchical Models: Cambridge University Press.

Gibson LA, Wilson BA, Cahill DM, and Hill J. 2004. Spatial prediction of rufous bristlebird habitat in a coastal heathland: a GIS-based approach. Journal of Applied Ecology 41:213-223.

Greenacre MJ. 1984. Theory and applications of correspondence analysis. London: Academic Press.

Hill MO, and Gauch HG. 1980. Detrended correspondence analysis: An improved ordination technique. Plant Ecology 42:47-58.

Hurlbert SH. 1984. Pseudoreplication and the Design of Ecological Field Experiments. Ecological Monographs 54:187-211. 
Ihm P, and Van Groenewoud H. 1984. Correspondence analysis and Gaussian ordination. COMPSTAT Lectures 3:5-60.

Ives AR, and Helmus MR. 2011. Generalized linear mixed models for phylogenetic analyses of community structure. Ecological Monographs 81:511-525.

Jamil T, Ozinga WA, Kleyer M, and ter Braak CJF. 2012. Selecting traits that explain species-environment relationships: a generalized linear mixed model approach. Journal of Vegetation Science:10.1111/j.1654-1103.2012.12036.x.

Jongman RHG, ter Braak CJF, and van Tongeren OFR. 1995. Data analysis in community and landscape ecology. Cambridge: Cambridge University Press.

Kruk C, Peeters E, Van Nes EH, Huszar VLM, Costa LS, and Scheffer M. 2011. Phytoplankton community composition can be predicted best in terms of morphological groups. Limnology and Oceanography 56:110-118.

Oksanen J, Blanchet FG, Kindt R, Legendre P, O'Hara RB, Simpson GL, Solymos P, Stevens MHH, and Wagner H. 2011. vegan: Community Ecology Package. R package version 1.17-12. http://CRANR-projectorg/package=vegan .

Oksanen J, Läärä E, Tolonen K, and Warner BG. 2001. Confidence Intervals for the Optimum in the Gaussian Response Function. Ecology 82:1191-1197.

Palmer MW, and Dixon PM. 1990. Small-Scale Environmental Heterogeneity and the Analysis of Species Distributions along Gradients. Journal of Vegetation Science 1:57-65.

Pollock LJ, Morris WK, and Vesk PA. 2012. The role of functional traits in species distributions revealed through a hierarchical model. Ecography 35:716-725.

Rue H, and Held L. 2005 Gaussian Markov Random Fields: Theory and Applications: London: Chapman and Hall-CRC Press. 
ter Braak CJF. 1985. Correspondence analysis of incidence and abundance data: properties in terms of a unimodal response model. Biometrics 41:859-873.

ter Braak CJF. 1986. Canonical correspondence analysis: a new eigenvector technique for multivariate direct gradient analysis. Ecology 67:1167-1179.

ter Braak CJF. 1987. Unimodal models to relate species to environment: DLO-Agricultural Mathematics Group, 1996: Wageningen, the Netherlands.

ter Braak CJF, and Looman CWN. 1986. Weighted averaging, logistic regression and the Gaussian response model. Vegetatio 65:3-11.

ter Braak CJF, and Prentice IC. 1988. A theory of gradient analysis. Advances in Ecological Research 18:271-317.

ter Braak CJF, and Prentice IC. 2004. A theory of gradient analysis. Advances in Ecological Research 34:235-282.

ter Braak CJF, and Smilauer P. 1998. CANOCO Reference Manual and User's Guide to Canoco for Windows: Software for Canonical Community Ordination (version 4). Ithaca, NY, USA: Microcomputer Power.

ter Braak CJF, and Verdonschot PFM. 1995. Canonical correspondence analysis and related multivariate methods in aquatic ecology. Aquatic Sciences 57:255-289.

Thompson R, Cullis B, Smith A, and Gilmour A. 2003. A sparse implementation of the average information algorithm for factor analytic and reduced rank variance models. Australian \& New Zealand Journal of Statistics 45:445-459.

Verbyla AP, Eckermann PJ, Thompson R, and Cullis BR. 2003. The analysis of quantitative trait loci in multi-environment trials using a multiplicative mixed model. Australian Journal of Agricultural Research 54:1395-1408.

Walker SC, and Jackson DA. 2011. Random-effects ordination: describing and predicting multivariate correlations and co-occurrences. Ecological Monographs 81:635-663. 
Whittaker RH. 1960. Vegetation of the Siskiyou Mountains, Oregon and California. Ecological Monographs 30:279-338.

Whittaker RH. 1967. Gradient analysis of vegetation. Biological Reviews of the Cambridge Philosophical Society 49:207-264.

Zhu M, Hastie TJ, and Walther G. 2005. Constrained ordination analysis with flexible response functions. Ecological Modelling 187:524-536.

Zuur AF, Ieno EN, Walker N, Saveliev AA, and Smith GM. 2009. Mixed Effects Models and Extensions in Ecology with R: Springer.

\section{Appendix}

This Appendix shows that similarity between the Gaussian logit models and GLMMs extends beyond the Gaussian logistic curve. The Gaussian logistic model can be extended to two environmental variables as follows (ter Braak \& Prentice 1988)

$$
\operatorname{logit}\left(p_{i j}\right)=a_{j}-\frac{1}{2}\left(d_{1}\left(x_{i 1}-u_{1 j}\right)^{2}+d_{2}\left(x_{i 2}-u_{2 j}\right)^{2}-2 d_{12 j}\left(x_{i 1}-u_{1 j}\right)\left(x_{i 2}-u_{2 j}\right)\right) .
$$

where $d$ 's are precision parameters, in the context of the bivariate normal distribution (Rue \& Held 2005 ). By setting

$$
\alpha_{j}=a_{j}-\frac{1}{2}\left(d_{1} u_{1 j}^{2}+d_{2} u_{2 j}^{2}-2 d_{12 j} u_{1 j} u_{2 j}\right),
$$

$$
\beta_{1 j}=d_{1} u_{1 j}-d_{12 j} u_{2 j},
$$

$$
\beta_{2 j}=d_{2} u_{2 j}-d_{12 j} u_{1 j},
$$


$390 \quad \beta_{3 j}=d_{12 j}$,

391 and

$392 \quad \gamma_{i}=\frac{-1}{2}\left(d_{1} x_{i 1}^{2}+d_{2} x_{i 2}^{2}\right)$

393 we can write

$394 \operatorname{logit}\left(p_{i j}\right)=\alpha_{j}+\beta_{1 j} x_{i 1}+\beta_{2 j} x_{i 2}+\beta_{3 j} x_{i 1} x_{i 2}+\gamma_{i}$.

395 Here $\beta_{3 \mathrm{j}}$ are random effects for interactions. If the 'co-precisions' are equal ( $d_{12 \mathrm{j}}=d_{12}$ )

396 the term $\beta_{3 j} x_{i 1} x_{i 2}$ can be subsumed in to the site effects $Y_{i}$ and the model can do

397 without interactions. The $\gamma_{i}$ account for the quadratic term arising from the Gaussian

398 (logit) model. Extension to more than two environmental variables is immediate. In

399 conclusion, up to distributional assumptions, the GLMM can be interpreted as a Gaussian

400 logistic model with equal tolerances for the species.

401 


\section{Figure 1}

Simulated unimodal response (occurrence probability $p_{i j}$ of a species at a site) against environmental variable $\mathrm{x}$ for a selection of species.

For left to right: three tolerances ( $t=0.5,1$ and 4$)$.
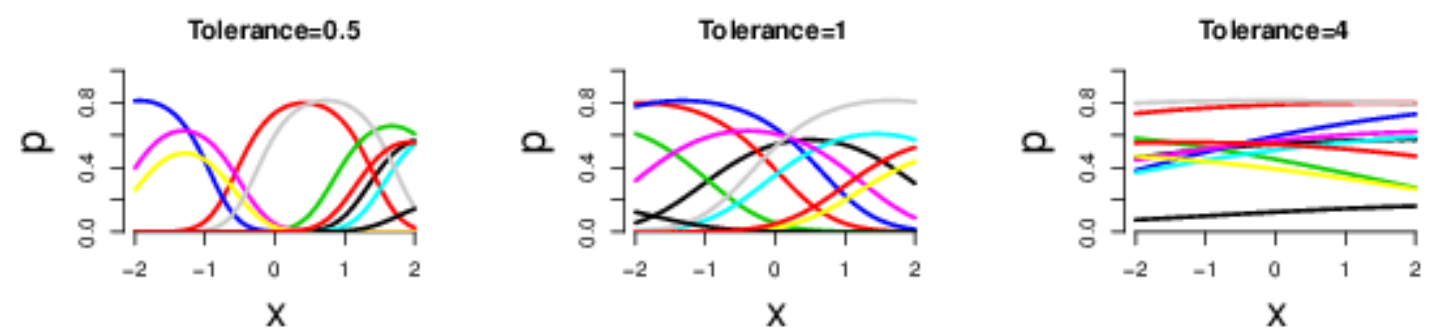


\section{Figure 2}

Simulated data: site effects as estimated by GLMM based on equation (1) plotted against environmental variable $\mathrm{x}$. A quadratic relationship indicates a unimodal response.

A quadratic relationship indicates a unimodal response. Rows: example series 1-3, columns: parameter varied.
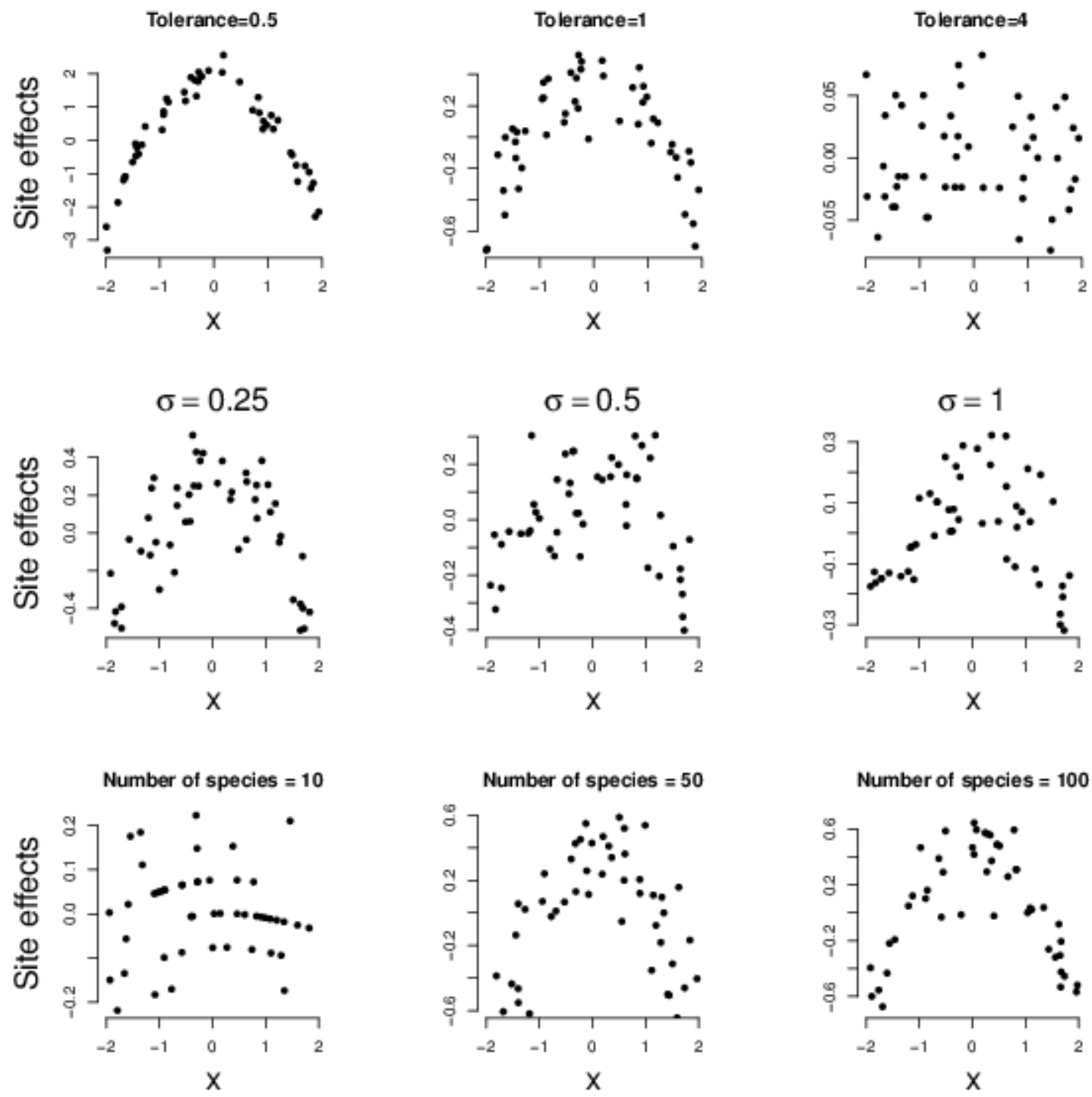


\section{Figure 3}

Simulated data: GLMM random slopes of equation (1) against the true optima of the Gaussian logistic curve of equation (2).

Rows: example series 1-3, columns: parameter varied. In the two bottom rows the (median) tolerance is 1 .
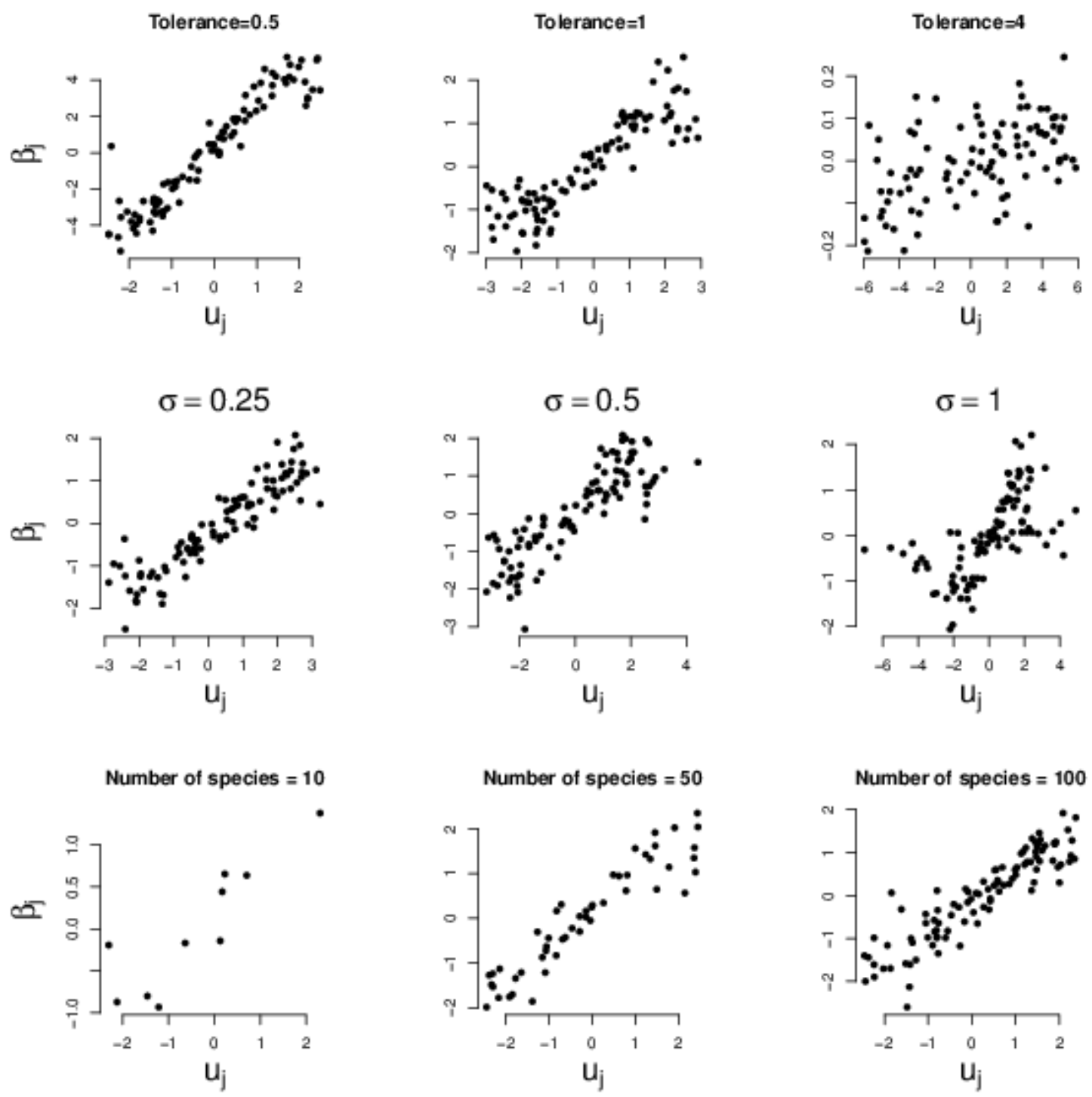


\section{Figure 4}

Real data: site effects (top) and the GLMM random slopes (bottom) plotted against the environmental variable $x$ and the optima of the Gaussian logistic curve as estimated by GLM, respectively.
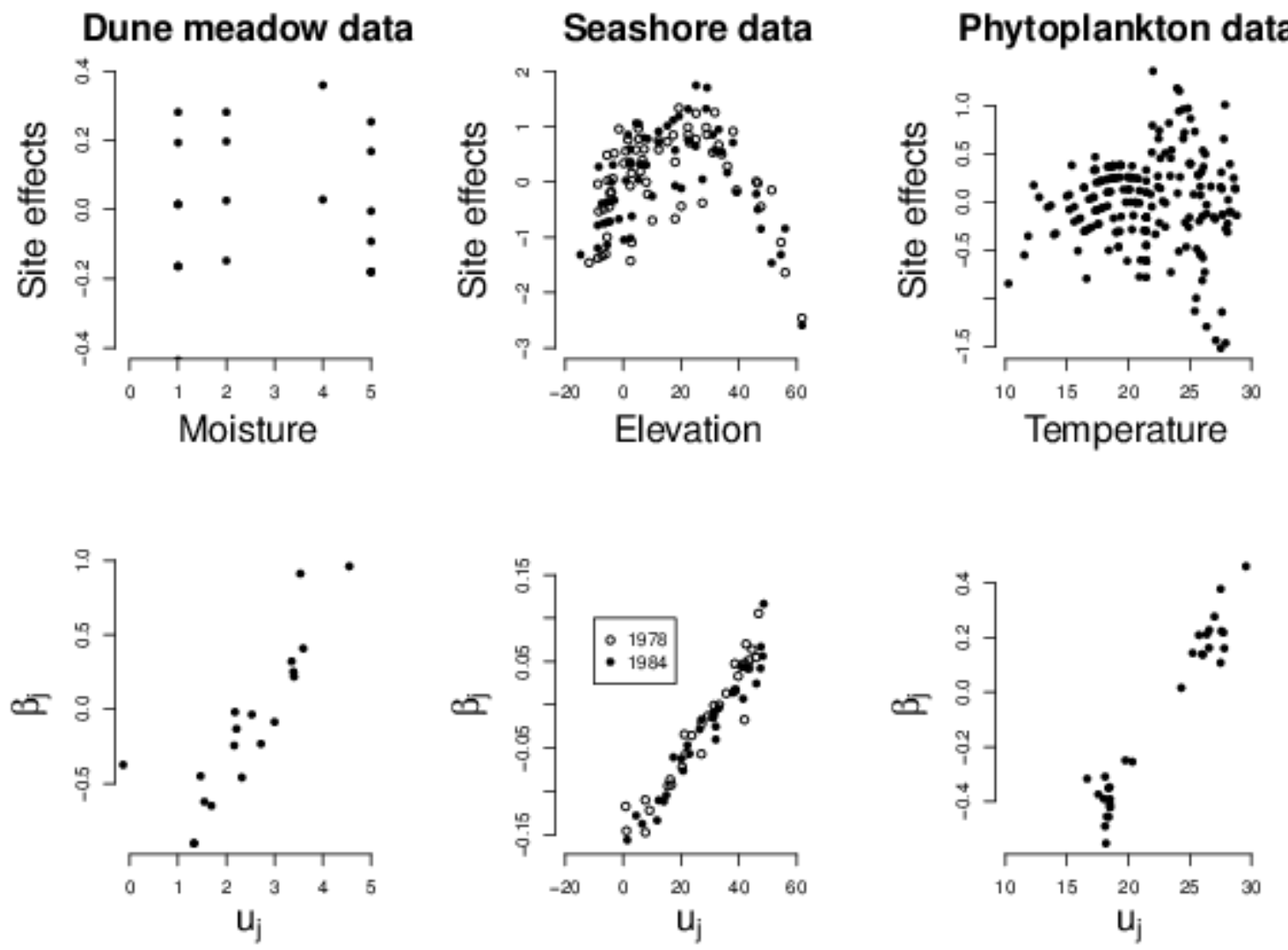